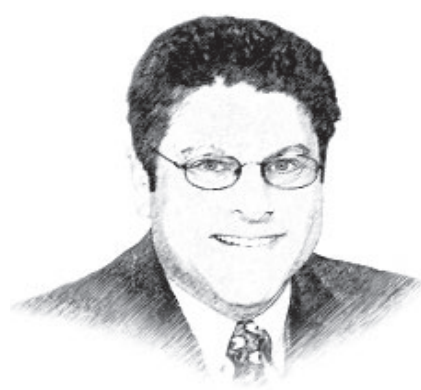

\title{
Double the reasons for giving the flu vaccine in 2020
}

Regular readers of the Journal can anticipate an annual fall article related to the flu vaccine or, at the least, be unsurprised by its publication. This year I asked Dr. Sherif Mossad, one of our infectious disease consultants with special expertise in respiratory viruses, to specifically address the potential relationship between the annual influenza vaccine and the ongoing COVID-19 pandemic. On page 651 of this issue, you will find his thoughtful response.

I always learn from Sherif. In his article he notes a fascinating (prepublication) observation from Brazil ${ }^{1}$ : patients who had received their 2020 influenza vaccination and who contracted SARS-CoV-2 fared better in several ways than those infected with the SARS-CoV-2 virus who had not been vaccinated. They were less likely to develop severe respiratory disease or die-a striking observation with obvious implications for all of us as we get ready for flu season.

The immunobiology underlying this observation, which hopefully is true, is not clear to me. This was an observational, not a prospective, randomized study. Hence there is the significant potential for bias due to potential specific reasons for giving the influenza vaccine to some but not all patients. The authors went to great lengths to limit this potential bias in their analysis. More than 90,000 patients were studied; final survival outcome data were available for 67,000, and recorded vaccination status data for more than 36,000 (about 40\%). Outcome benefit was most pronounced in patients over age 60. Most intriguing is the observation that patients who received the influenza vaccine while symptomatic from COVID-19 still received significant benefit in terms of pulmonary and survival outcome (odds ratio for mortality $0.73,95 \%$ confidence interval 0.58-0.91). This rapid effect argues against the boosting of an antibody that cross-reacts between the two viruses (adaptive immunity) being the mechanism, and rather favors a boosting of the innate immune response (less-specific pathogen recognition or perhaps stimulation of interferon generation). This is consistent with cross-agent protection from some other vaccines reported in the past.

I have already added this discussion to my dialogue with patients who are hesitant to get the flu vaccine this year. "This year in particular," I say, "is not the year to avoid getting vaccinated." I emphasize the similarity in symptoms between early influenza and COVID-19, which could lead to enormous angst and implications regarding quarantine from family, work, and school. Receiving the flu vaccine should lessen the likelihood of this happening. I think it has helped my case in promoting vaccination.

In a second vaccine-related paper in this issue of the Journal, Day et al (page 695) discuss the increasingly common question of which vaccines can and should be given to patients receiving immunosuppressive therapy. As use of "biologics" has proliferated in the successful treatment of more diseases, internists and other primary care providers in the office often face this question. New information indicates that patients receiving the Janus kinase inhibitors are more prone to experience outbreaks of herpes zoster, raising the imperative for considering administration of Shingrix, a

doi:10.3949/ccjm.87b.11020 
recombinant adjuvanted "dead" zoster vaccine, to these patients before or concurrent with starting one of these medications. Additionally, there are recent studies suggesting that the efficacy of the normal-strength influenza vaccine given to patients receiving methotrexate can be increased by withholding the methotrexate for 2 weeks after vaccination (or by using the higher-dose vaccine intended for patients over age 65). This is information relevant to both subspecialists and primary care providers.

I believe that vaccinations contribute to improving the public health. And while we all hopefully await the arrival of an appropriately evaluated, safe, and effective vaccine against COVID-19 endorsed by the National Institute of Allergy and Infectious Diseases, we need to do the best we can to limit the impact of other vaccination-preventable infectious diseases, as well as the spread of the current pandemic.

And thank you, Dr. Anthony Fauci.

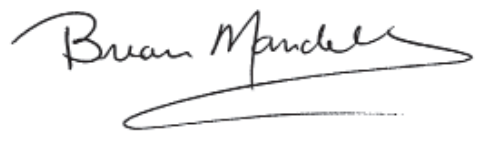

Brian F. Mandell, MD, PhD Editor in Chief

1. Fink $\mathbf{G}$, Orlova-Fink $\mathbf{N}$, Schindler $\mathbf{T}$, et al. Inactivated trivalent influenza vaccine is associated with lower mortality among Covid-19 patients in Brazil. MedRxiv 2020 Jul 1. doi: https://doi.org/10.1101/2020.06.29.20142505 